\section{AN AUDIT OF MANAGEMENT OF NEUTROPENIC AND NON-NEUTROPENIC FEVER IN HAEMATOLOGY-ONCOLOGY PATIENTS BY A UK TERTIARY PAEDIATRIC HOSPITAL}

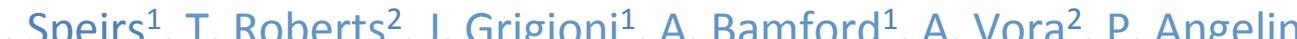

Great Ormond Street Hospital, Paediatric Infectious Diseases, London, United Kingdom.

${ }^{2}$ Great Ormond Street Hospital, Paediatric Haematology, London, United Kingdom.

${ }^{3}$ The Royal Marsden Hospital, Paediatric Oncology, London, United Kingdom

Background

- In children receiving chemotherapy for malignancy, febrile neutropenia is a common complication

- Management guidelines exist for use within our tertiary care hospital and for our shared care centres which are based on the National Institute of Clinical Excellence guidelines (2012)

Aims

- To perform a prospective audit of haematology-oncology patients with neutropenic and nonneutropenic fever admitted to or discussed with our centre

- To assess adherence to current management guideline, determine outcomes and identify areas of our guideline requiring modification.

Methods

- All patients in August 2017 who were discussed or managed in our centre (via electronic notes)

- Information was collected on age, diagnosis, level of neutropenia, clinical presentation, antimicrobials used, investigations performed and microbiology results.

- Project approval was granted by the hospital audit department

\begin{tabular}{|c|c|}
\hline Figure 1: Underlying Diagnosis $\begin{array}{lll}1 & 21 \\
6 & & \end{array}$ & $\begin{array}{l}\text { Acute Lymphoblastic Leukaemia } \\
\square \text { Neuroblastoma } \\
\text { Acute Myeloid Leukaemia } \\
\square \text { Brain Tumor } \\
\square \text { Germ Cell Tumour } \\
\square \text { Rhabdoid Tumour } \\
\square \text { Rhabdomyosarcoma } \\
\text { Wilm's Tumour }\end{array}$ \\
\hline
\end{tabular}

Results

- Fifty-one patients were identified (25 inpatients, 26 telephone advice)

- There were 3 deaths with one due to gram-negative sepsis.

- Symptoms documented in $53 \%$ and only $10 \%$ had clear evidence of risk stratification.

- As first-line treatment, 57\% received the recommended piperacillin-tazobactam and aminoglycoside or ciprofloxacin.

- Six patients received meropenem first line, $50 \%$ of these were due to piperacillin-tazobactam resistant bacteria in stool identified on routine screening (local trust protocol)

- Positive blood cultures were identified in $12 \%$, most commonly coagulase negative staphylococcus.

- Overall, $67 \%$ of patients were managed as per guideline (see figure 3 )

- Common reasons for guideline deviation were prolonged aminoglycoside use and early addition of antifungals or teicoplanin/vancomycin.

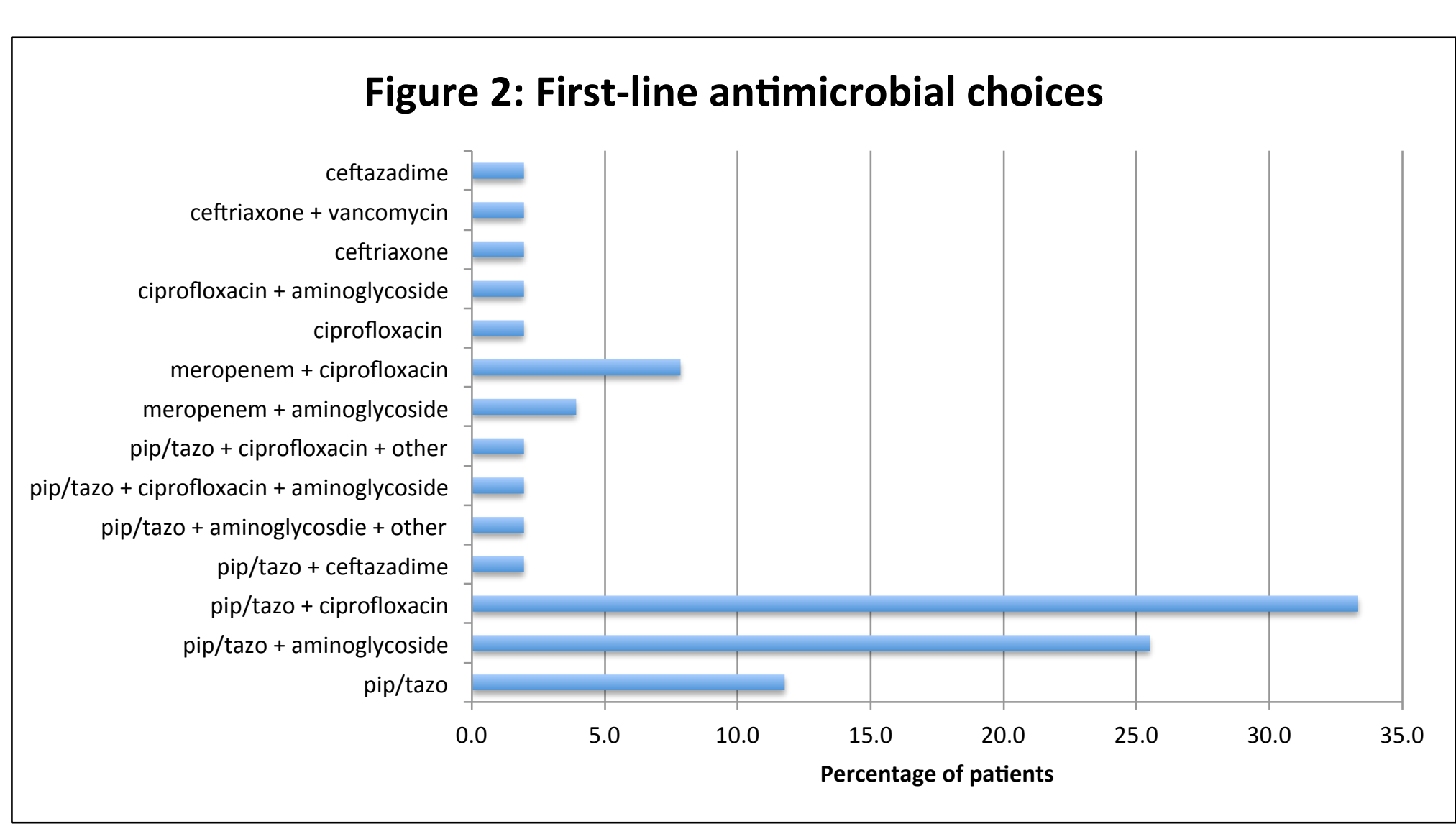

Figure 3: Adherence to guideline recommendations

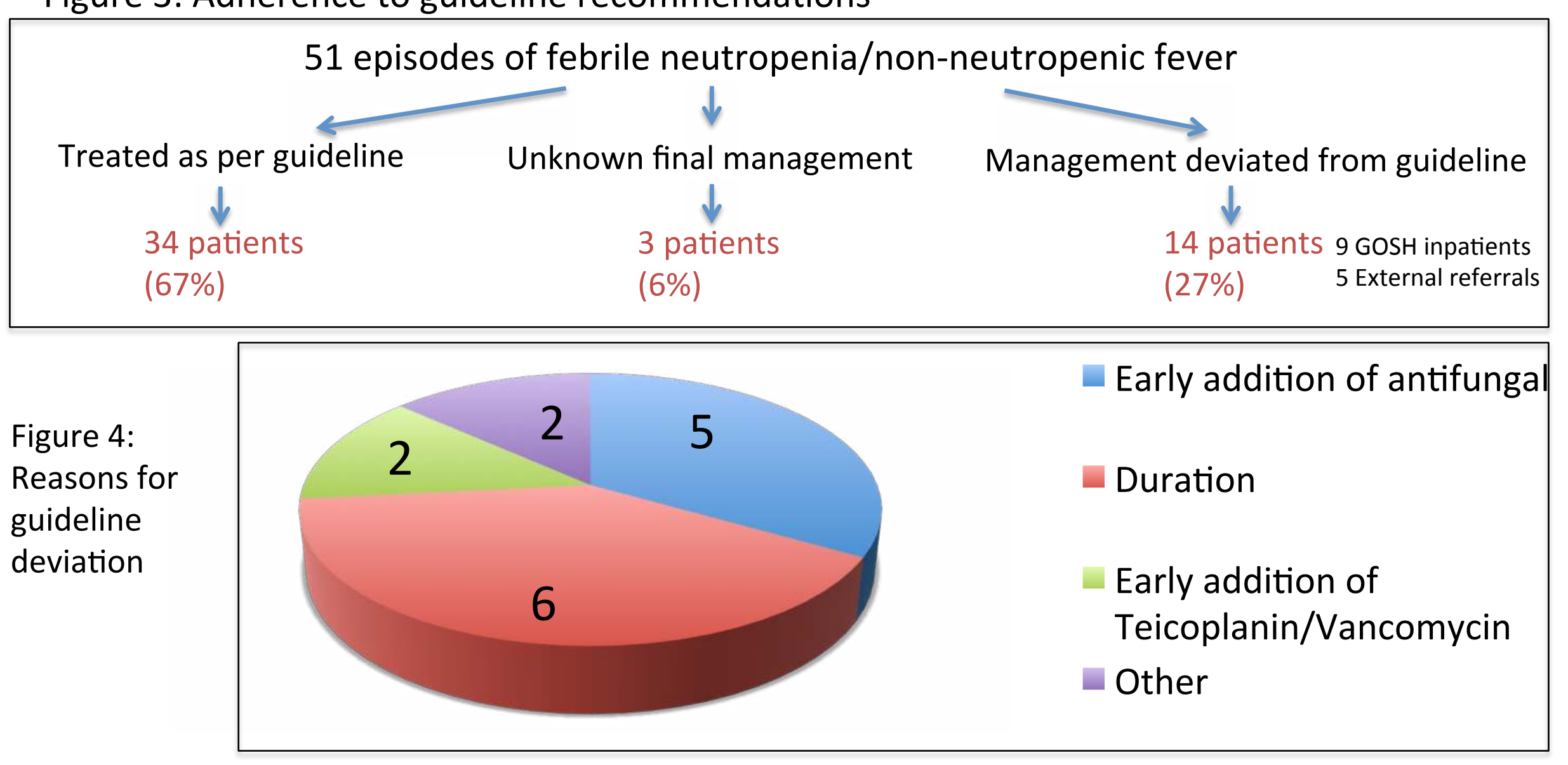

Conclusions

- Existing guidelines are not always followed in our centre. Reasons for this could include a lack of clarity in the guidance or individual patient complexity, resulting in treatment plans which deviate from the guideline.

- Education is required on improving clinical assessment and risk stratification documentation.

Ongoing work:

- $\quad$ Revision of local shared care guidelines to make them more up to date with current guidance and more user friendly

Education about management of febrile neutropenia at shared care study day Re-audit once new guidelines are circulated 